\title{
Cool and warm dust emission from M 33 (HerM33es) $^{\star}$
}

\author{
E. M. Xilouris ${ }^{1}$, F. S. Tabatabaei ${ }^{2}$, M. Boquien ${ }^{3}$, C. Kramer $^{4}$, C. Buchbender ${ }^{4}$, F. Bertoldi ${ }^{5}$, S. Anderl ${ }^{5}$, J. Braine ${ }^{6}$, \\ S. Verley ${ }^{7}$, M. Relaño ${ }^{7}$, G. Quintana-Lacaci ${ }^{4}$, S. Akras ${ }^{8}$, R. Beck ${ }^{9}$, D. Calzetti ${ }^{10}$, F. Combes ${ }^{11}$, M. Gonzalez ${ }^{4}$, \\ P. Gratier ${ }^{12}$, C. Henkel ${ }^{9}{ }^{13}$, F. Israel ${ }^{14}$, B. Koribalski ${ }^{15}$, S. Lord $^{16}$, B. Mookerjea ${ }^{17}$, E. Rosolowsky ${ }^{18}$, G. Stacey ${ }^{19}$, \\ R. P. J. Tilanus ${ }^{20,21}$, F. van der $\operatorname{Tak}^{22}$, and P. van der $\operatorname{Werf}^{14,23}$
}

(Affiliations can be found after the references)

Received 27 March 2012 / Accepted 3 May 2012

\begin{abstract}
In the framework of the open-time key program "Herschel M 33 extended survey (HerM33es)", we study the far-infrared emission from the nearby spiral galaxy M 33 in order to investigate the physical properties of the dust such as its temperature and luminosity density across the galaxy. Taking advantage of the unique wavelength coverage $(100,160,250,350$, and $500 \mu \mathrm{m})$ of the Herschel Space Observatory and complementing our dataset with Spitzer-IRAC 5.8 and $8 \mu \mathrm{m}$ and Spitzer-MIPS 24 and $70 \mu \mathrm{m}$ data, we construct temperature and luminosity density maps by fitting two modified blackbodies of a fixed emissivity index of 1.5. We find that the "cool" dust grains are heated to temperatures of between $11 \mathrm{~K}$ and $28 \mathrm{~K}$, with the lowest temperatures being found in the outskirts of the galaxy and the highest ones both at the center and in the bright HII regions. The infrared/submillimeter total luminosity $(5-1000 \mu \mathrm{m})$ is estimated to be $1.9 \times 10^{9}{ }_{-4.4 \times 10^{8}}^{+4.0 \times 10^{8}} L_{\odot}$. Fifty-nine percent of the total infrared/submillimeter luminosity of the galaxy is produced by the "cool" dust grains $(\sim 15 \mathrm{~K})$, while the remaining $41 \%$ is produced by "warm" dust grains $(\sim 55 \mathrm{~K})$. The ratio of the cool-to-warm dust luminosity is close to unity (within the computed uncertainties), throughout the galaxy, with the luminosity of the cool dust being slightly higher at the center than the outer parts of the galaxy. Decomposing the emission of the dust into two components (one emitted by the diffuse disk of the galaxy and one emitted by the spiral arms), we find that the fraction of the emission from the disk in the mid-infrared $(24 \mu \mathrm{m})$ is $21 \%$, while it gradually rises up to $57 \%$ in the submillimeter $(500 \mu \mathrm{m})$. We find that the bulk of the luminosity comes from the spiral arm network that produces $70 \%$ of the total luminosity of the galaxy with the rest coming from the diffuse dust disk. The "cool" dust inside the disk is heated to temperatures in a narrow range between $18 \mathrm{~K}$ and $15 \mathrm{~K}$ (going from the center to the outer parts of the galaxy).
\end{abstract}

Key words. Local Group - galaxies: spiral - galaxies: ISM

\section{Introduction}

Studies of the dust content and the properties of the interstellar medium (ISM) within nearby galaxies have been a major topic of research since the launch of the Infrared Astronomical Satellite (IRAS). The IRAS measurements performed at $60 \mu \mathrm{m}$ and $100 \mu \mathrm{m}$, being most sensitive to the emission from the warm dust $(\sim 45 \mathrm{~K})$ peaking at these wavelengths, provided a unique database for studying this fraction of the total ISM (e.g. Young et al. 1996; Devereux \& Young 1990). With the advent of the Infrared Space Observatory (ISO) and the Spitzer Space Telescope, observing at wavelengths longer than $100 \mu \mathrm{m}$, as well as the submillimeter (submm) observations of the James Clerk Maxwell Telescope (JCMT), it became evident that the bulk of the dust grains reside in a cooler (below $20 \mathrm{~K}$ ) component that makes the most significant contribution to the total far-infrared (FIR) and submm emission of a galaxy. Although the existence of the warm and cool components is now wellestablished, spatial information on the distribution of their emission within galaxies is still poorly known. Galaxies showing prominent spiral structure at optical wavelengths have strong dusty spiral arms as well as dust material diffusely distributed throughout the galaxy revealing itself in the inter-arm regions (e.g., Haas et al. 1998; Gordon et al. 2006; Hippelein et al. 2003). A simplistic, but nevertheless elegant, way of distinguishing

\footnotetext{
* Herschel is an ESA space observatory with science instruments provided by European-led Principal Investigator consortia and with important participation from NASA.
}

between the dust diffusely distributed throughout the galaxy and the dust situated inside the spiral arms is to assume that the spiral arm network is superimposed on an axisymmetric dusty disk (e.g. Misiriotis et al. 2000; Meijerink et al. 2005). Herschel Space Observatory (Pilbratt et al. 2010) with its unique wavelength coverage, resolving power, and sensitivity is an excellent provider of data for these studies.

Owing to their proximity, the galaxies in the Local Group are ideal systems for carrying out high spatial resolution studies of the ISM. As an Sc spiral galaxy at a distance of $840 \mathrm{kpc}$ (Freedman et al. 1991), M 33 is an ideal candidate for the present analysis. Previously mapped by ISO at 60,100 , and $170 \mu \mathrm{m}$ at a moderate resolution, M 33 reveals a spiral structure with a large number of distinct sources, as well as a diffuse extended component (Hippelein et al. 2003). In the same study, spectral energy distributions (SEDs) constructed for the central part of the galaxy as well as the interarm regions and prominent HII regions, reveal typical temperatures of $T_{\mathrm{w}} \sim 46 \mathrm{~K}$ for the warm dust and $T_{\mathrm{c}} \sim 17 \mathrm{~K}$ for the cool dust component. Performing a multi-scale study of the infrared (IR) emission of M33 using Spitzer-MIPS data and a wavelet analysis technique, Tabatabaei et al. (2007a) concluded that most of the $24 \mu \mathrm{m}$ and $70 \mu \mathrm{m}$ emission emerge from bright HII regions and star-forming complexes, while the $160 \mu \mathrm{m}$ emission traces both compact and diffuse emission throughout the galaxy. Using intensity maps at $70 \mu \mathrm{m}$ and $160 \mu \mathrm{m}$, Tabatabaei et al. (2007b) constructed temperature maps that illustrated the variations in the temperature of between $19 \mathrm{~K}$ and $28 \mathrm{~K}$, while similar conclusions were reached 

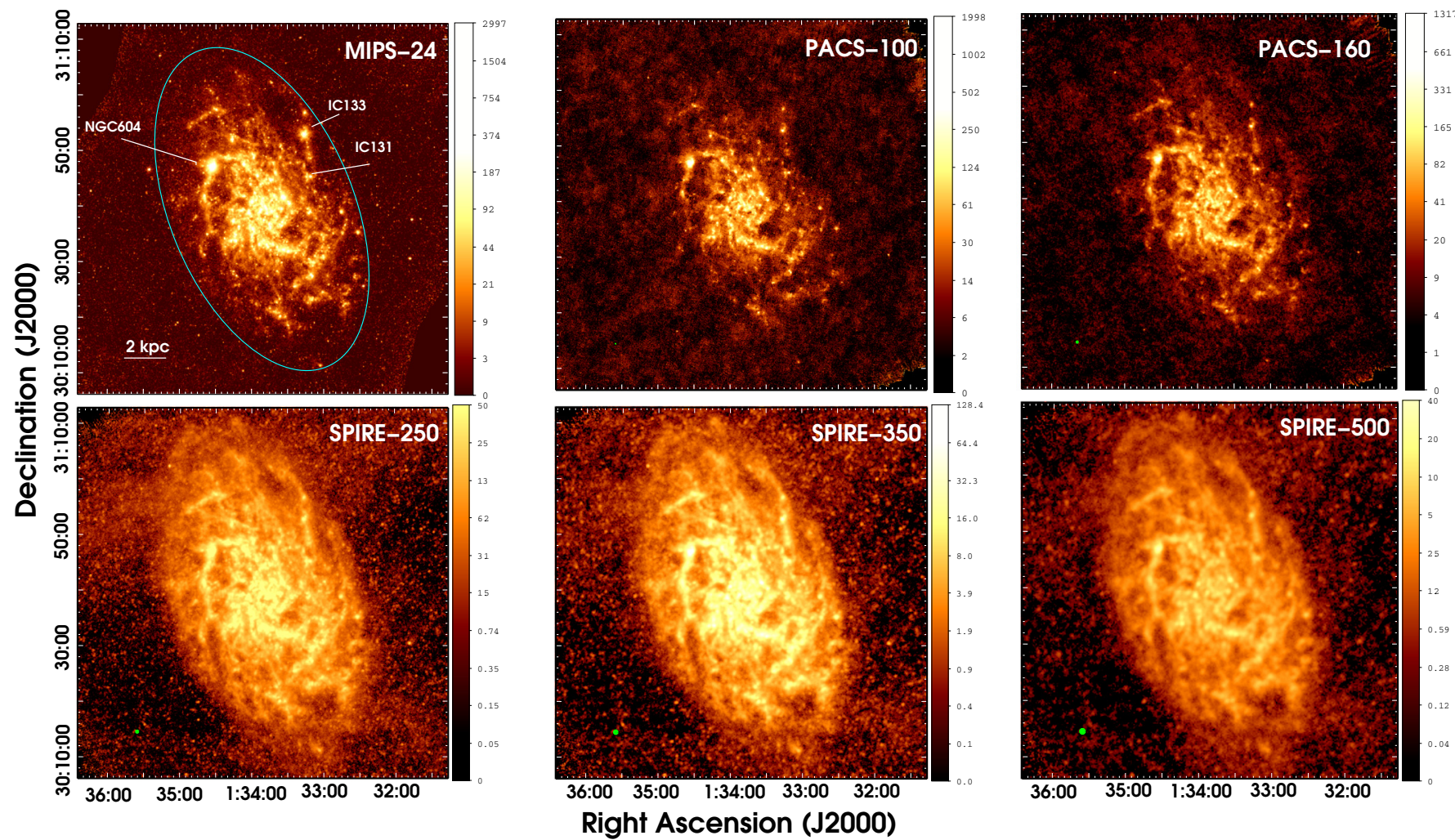

Fig. 1. Spitzer MIPS-24 $\mu \mathrm{m}$ (upper left panel), Herschel PACS-100 and $160 \mu \mathrm{m}$ (upper middle and right panels, respectively) and SPIRE-250, 350 , and $500 \mu \mathrm{m}$ (lower left, middle and right panels, respectively). All maps are at their original resolution, and the FWHM of the beams (6", $6.7^{\prime \prime} \times 6.9^{\prime \prime}, 10.7^{\prime \prime} \times 11.1^{\prime \prime}, 18.3^{\prime \prime} \times 17.0^{\prime \prime}, 24.7^{\prime \prime} \times 23.2^{\prime \prime}$, and $37.0^{\prime \prime} \times 33.4^{\prime \prime}$ for $24,100,160,250,350$, and $500 \mu$ m respectively) are indicated as green circles at the bottom-left part in each panel. In the MIPS-24 $\mu \mathrm{m}$ map, three of the brightest HII regions are labeled for reference. The outer ellipse shows the $B$-band $\mathrm{D}_{25}$ (semi-major axis length $=7.5 \mathrm{kpc}$; Paturel et al. 2003) extent of the galaxy. The brightness scale is given in units of $\mathrm{MJy} \mathrm{sr}^{-1}$.

by Verley et al. (2008) by constructing the dust temperature variations as a function of radius from the center.

The recently launched Herschel Space Observatory offers the possibility to study M33 at FIR and submm wavelengths in more detail than possible before. The PACS (Poglitsch et al. 2010) and SPIRE (Griffin et al. 2010) instruments combined allow us to produce images over the wavelength range between $70 \mu \mathrm{m}$ and $500 \mu \mathrm{m}$ with unprecedented sensitivity and superior resolution. In this paper, we use PACS $(100 \mu \mathrm{m}$ and $160 \mu \mathrm{m})$ and SPIRE $(250 \mu \mathrm{m}, 350 \mu \mathrm{m}, 500 \mu \mathrm{m})$ imaging, taken as part of the Herschel M 33 extended survey (HerM33es; Kramer et al. 2010), an open-time key program, along with a two-component modified blackbody model in order to improve our understanding of the nature of the continuum emission of M33. With this approach, we hope to shed some light on the temperature and luminosity distributions of M33, being aware of the simplistic nature of the model that is used. A more realistic analysis (within the framework of the HerM33es open-time key program) is presented elsewhere (Rosolowsky et al., in prep). In Sect. 2, we present the observations and the data reduction techniques that we use, Sect. 3 gives a brief description of the morphology of the galaxy at the Herschel wavelengths, Sect. 4 describes the modeling of the data, and the results of our study are presented in Sect. 5 and discussed in Sect. 6. We finally present our conclusions in Sect. 7.

\section{Observations and data reduction}

The Local Group galaxy M33 was observed on 7 and 8 January 2010 with PACS and SPIRE covering a field of $70^{\prime} \times 70^{\prime}$. Observations were made using the PACS and SPIRE parallel mode with a scanning speed of $20^{\prime \prime} / \mathrm{s}$ providing simultaneous observations at $100 \mu \mathrm{m}$ and $160 \mu \mathrm{m}$, as well as at $250 \mu \mathrm{m}$, $350 \mu \mathrm{m}$, and $500 \mu \mathrm{m}$. The PACS maps presented in this paper were reduced using the scanamorphos algorithm (Roussel 2012) discussed in detail in Boquien et al. (2011) and in Rosolowsky et al. (in prep.). The SPIRE maps were reduced using HIPE 7.0 (Ott 2010) and the SPIRE calibration tree v. 7.0. SPIRE characteristics (point spread function, beam area, bandpass transmission curves, and correction factors for extended emission) were taken from the SPIRE Observers' Manual (v2.4 2011). A baseline algorithm (Bendo et al. 2010) was applied to every scan of the maps in order to correct for offsets between the detector timelines and remove residual baseline signals. Finally, the maps were created using a "naive" mapping projection. Regarding the errors in the PACS and SPIRE photometry, we adopted a conservative $15 \%$ calibration uncertainty for extended emission (Boquien et al. 2011; SPIRE Observers’ Manual, v2.4 2011).

In addition to the Herschel maps, we also used the publicly available mid-infrared (MIR) maps at $5.8 \mu \mathrm{m}$ and $8 \mu \mathrm{m}$ obtained with the IRAC instrument, and the $24 \mu \mathrm{m}$ and $70 \mu \mathrm{m}$ obtained with the MIPS instrument on-board the Spitzer Space Observatory. To account for the stellar pollution in the $5.8 \mu \mathrm{m}$ and $8 \mu \mathrm{m}$ fluxes, we used the IRAC-3.6 $\mu \mathrm{m}$ data as a reference point assuming that fluxes at this waveband only trace stellar emission. This, however, is a crude approximation since dust contamination, especially in the bright HII regions, may be present in $3.6 \mu \mathrm{m}$. We then corrected the $5.8 \mu \mathrm{m}$ and $8 \mu \mathrm{m}$ maps by assuming a stellar light contamination dictated by the $3.6 \mu \mathrm{m}$ measurements scaled by a Rayleigh-Jeans law. 
E. M. Xilouris et al.: Cool and warm dust emission from M33 (HerM33es)

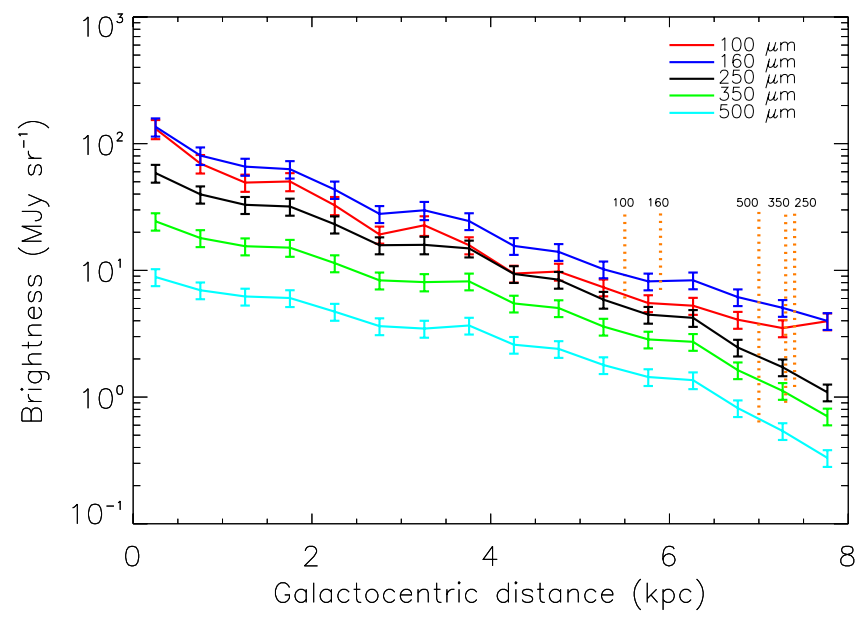

Fig. 2. Azimuthally averaged radial profiles, projected onto the major axis of M 33 for the $100,160,250,350$, and $500 \mu \mathrm{m}$ emission (red, blue, black, green, and cyan colors, respectively). The vertical dotted lines (in orange color) indicate the 3- $\sigma$ flux level threshold for each wavelength.

The maps used in our analysis had all been convolved to a common angular resolution (the resolution of the $500 \mu \mathrm{m}$ SPIRE image; approximately $40^{\prime \prime}$ ) by using the dedicated convolution kernels provided by Aniano et al. $(2011)^{1}$, and then projected onto the same grid (of a 10" pixel) and central position.

\section{Morphology}

Figure 1 shows the maps obtained at the five Herschel wavelengths $(100,160,250,350$, and $500 \mu \mathrm{m})$ as well as the $24 \mu \mathrm{m}$ map for comparison. In the $24 \mu \mathrm{m}$ map, we have also indicated the $B$-band $\mathrm{D}_{25}(7.5 \mathrm{kpc}$; Paturel et al. 2003) extent of the galaxy, as well as the three of the brightest HII regions. All maps are at their original resolution and the brightness scale is in $\mathrm{MJy} \mathrm{sr}^{-1}$.

We used the Herschel maps to spatially model the SED across the galaxy (see Sect. 4). All Herschel maps clearly show the spiral arm structure and a large number of distinct sources. In addition to the spiral structure, an underlying extended emission component can be recognized, particularly in the three SPIRE maps and even more clearly in the azimuthally averaged radial profiles presented in Fig. 2. These profiles correspond to average fluxes within successive elliptical rings of $500 \mathrm{pc}$ width and a minor-to-major axis ratio of 0.54 accounting for an inclination of 56 degrees (Regan \& Vogel 1994). The profiles are plotted all the way out to $8 \mathrm{kpc}$ with the orange vertical lines indicating the 3- $\sigma$ threshold in each band. We fitted to these profiles exponential functions (by minimizing the $\chi^{2}$ ), in order to derive the characteristic scalelength of the dust emission at each wavelength. We did this for the range of radii with fluxes above the 3- $\sigma$ threshold. We inferred scalelengths of $1.84 \pm 0.12 \mathrm{kpc}, 2.08 \pm 0.09 \mathrm{kpc}, 2.14 \pm 0.08 \mathrm{kpc}$, $2.50 \pm 0.12 \mathrm{kpc}$, and $3.04 \pm 0.15 \mathrm{kpc}$ for the $100 \mu \mathrm{m}, 160 \mu \mathrm{m}$, $250 \mu \mathrm{m}, 350 \mu \mathrm{m}$, and $500 \mu \mathrm{m}$ images, respectively. The scalelength that we derived for the $160 \mu \mathrm{m}$ emission using the Herschel-PACS data is in excellent agreement with the value obtained using the Spitzer-MIPS data $(1.99 \pm 0.02 \mathrm{kpc}$; Verley et al. 2009). The flatter dust distribution at longer wavelengths is indicative of a more extended distribution related to emission

\footnotetext{
${ }^{1}$ http://www . astro.princeton. edu/ ganiano/Kernels.html
}

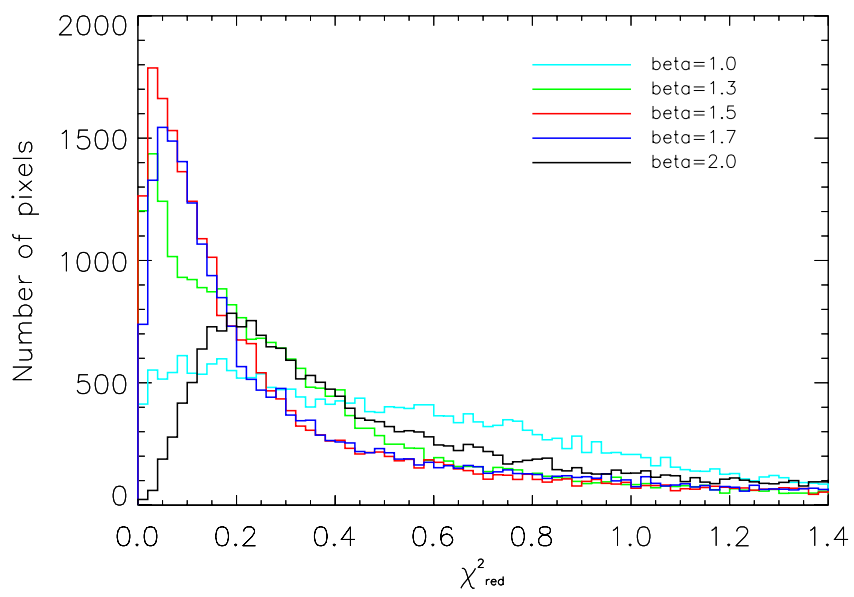

Fig. 3. Histogram of the $\chi_{\text {red }}^{2}$ values for the three models with $\beta=1,1.3$, $1.5,1.7$, and 2 (cyan, green, red, blue, and black line, respectively).

dominated by "cool" ( $\sim 15 \mathrm{~K})$ dust grains (see Sect. 6.1). Similar conclusions have been reached by others using ISO (e.g., Alton et al. 1998; Davies et al. 1999) and Herschel (e.g., Bendo et al. 2012) observations.

The spiral arms detected at all Herschel bands can be traced all the way to the center of the galaxy (to a $30 \mathrm{pc}$ scale; which is roughly the PACS $100 \mu \mathrm{m}$ resolution). Two main spiral arms, one to the north-east and one to the south-west, are the brightest large-scale features of the galaxy. The main spiral structure is also present at optical, near-infrared (NIR), and radio wavelengths (Block et al. 2007; Tabatabaei et al. 2007b). Apart from these two open spiral arms, our observations show numerous, apparently independent, long-arm spiral features in the outer regions of the galaxy, that have a smaller pitch angle. These features form a flocculent spiral structure, while in the NIR two arcs or wound-up spiral arms delineate another coherent pattern. M33 is not the only galaxy to have a flocculent spiral structure in young tracers, such as gas and young stars, superposed on a more grand-design spiral structure (e.g. Block et al. 1996). These branched multiple spirals could be random and independent wave packets triggered by local gravitational instabilities in the gas and stars (Binney \& Tremaine 1987) or they could result entirely from propagating star formation and differential rotation (Gerola \& Seiden 1978). In this case, gaseous instabilities trigger a first episode of star formation leading to supernova explosions and shell-like compressions in the ISM. This triggers additional star formation leading to a chain reaction in which aggregates of stars are created (see, e.g., Verley et al. 2010). The differential rotation of the galaxy, then, stretches these aggregates into spiral arcs. A characteristic picture of these violent processes is also evident in M33 in terms of a huge $(>1 \mathrm{kpc})$ shell-like feature that appears to break the south-west spiral arm (which is clearly evident in all Herschel maps of Fig. 1). A detailed analysis of the morphology of the gas and dust content of M 33 is presented in Combes et al. (2012) using power-spectrum analysis techniques.

This picture is in excellent agreement with other tracers of the ISM such as the atomic and the molecular hydrogen (Braine et al. 2010; Gratier et al. 2010, 2012). Many localized IR sources are well-detected in the Herschel bands. These sources are mainly HII regions situated in the spiral arms showing enhanced star-formation activity. A detailed analysis of the properties of these sources is presented in Verley et al. (2010) as well as in Boquien et al. (2010). 

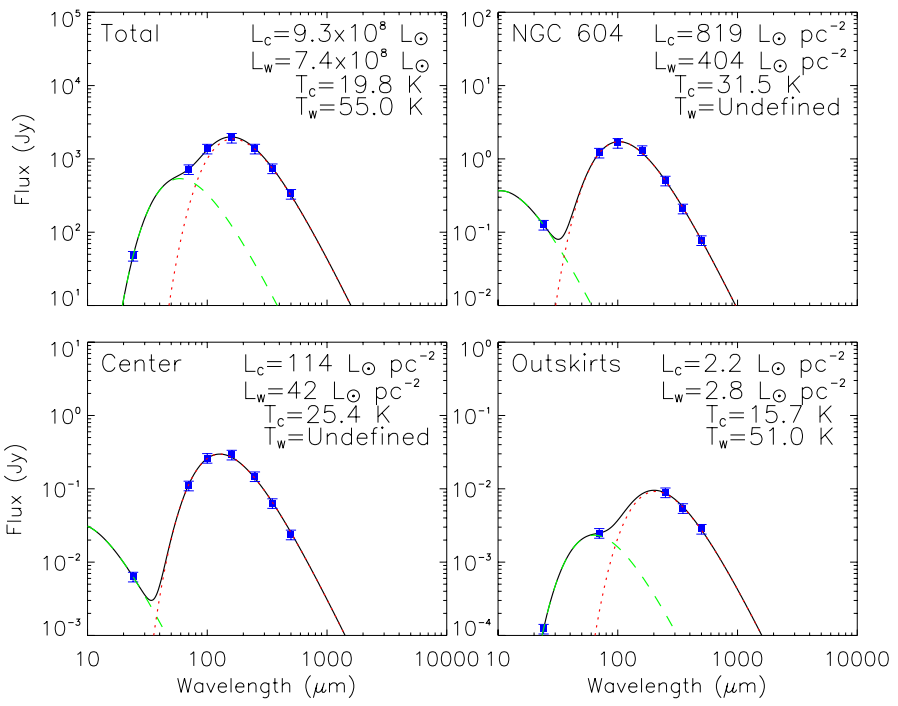

Fig. 4. Spectral energy distributions for the total galaxy (upper left panel) and a typical HII region (upper right - center of NGC 604), as well as the center of the galaxy (lower left panel) and the "diffuse" outer part of the galaxy (lower right panel). The emission was modeled with two modified blackbodies of $\beta=1.5$ and fitted to the MIPS 24 and $70 \mu \mathrm{m}$, PACS 100 and $160 \mu \mathrm{m}$, and SPIRE 250, 350, and $500 \mu \mathrm{m}$ observations. For the bright parts of the galaxy (around the center and in bright HII regions), it is evident that the specific model is unable to accurately describe the data giving unphysically high temperatures for the "warm" dust component. In the text, we describe two different and more reliable methods to derive the properties of the "warm" dust component (see Sect. 4.1 for more details).

\section{The model}

The unprecedented spatial resolution in the FIR and submm wavelengths obtained with Herschel allows us to study the dust grain properties on a very small scale throughout the galaxy. For this purpose, the dust SED was modeled assuming optically thin emission described by two modified blackbodies, one accounting for the "cool" dust emission, originating from the large grains being heated by the local and the diffuse galactic radiation field, and one accounting for the "warm" dust emission, originating from the grains being heated in dense and warm environments where star formation is taking place. According to this model, the emission at a given frequency $(v)$ is described by

$S_{v}=\frac{1}{\lambda^{\beta}}\left[N_{\mathrm{c}} B\left(v, T_{\mathrm{c}}\right)+N_{\mathrm{w}} B\left(v, T_{\mathrm{w}}\right)\right]$,

where $B(v, T)$ is the Planck function, and $T_{\mathrm{c}}$ and $T_{\mathrm{w}}$ are the dust temperatures for the "cool" and the "warm" dust component, respectively, $\beta$ is the emissivity index, and both $N_{\mathrm{c}}$ and $N_{\mathrm{w}}$ are normalization constants related to the dust column density in each of the "cool" and the "warm" components, respectively. To find the best-fit SED for each pixel on the map, our code minimizes the $\chi^{2}$ function $\left(\chi^{2}=\sum\left(\left(S_{v, \mathrm{obs}}-S_{v}\right) / \Delta S_{v \text {,obs }}\right)^{2}\right)$ using the Levenberg-Marquardt algorithm (Bevington \& Robinson 1992). The model was applied to the $24,70,100,160,250,350$, and $500 \mu \mathrm{m}$ maps regridded to a common resolution and pixel size (see Sect. 2) and for all pixels with intensities higher than three times the root mean square (rms) noise level. The rms noise level for the $\left(F W H M=40^{\prime \prime}\right.$ and pixel size $\left.=10^{\prime \prime}\right)$ maps that we used are $0.012,0.31,3.2,3.4,0.64,0.50$, and $0.32 \mathrm{mJy} \mathrm{pix}^{-1}$ for the $24,70,100,160,250,350$, and $500 \mu \mathrm{m}$ respectively. These values were obtained by performing the statistics in regions well outside the galaxy. To expand our modeling to fainter parts of

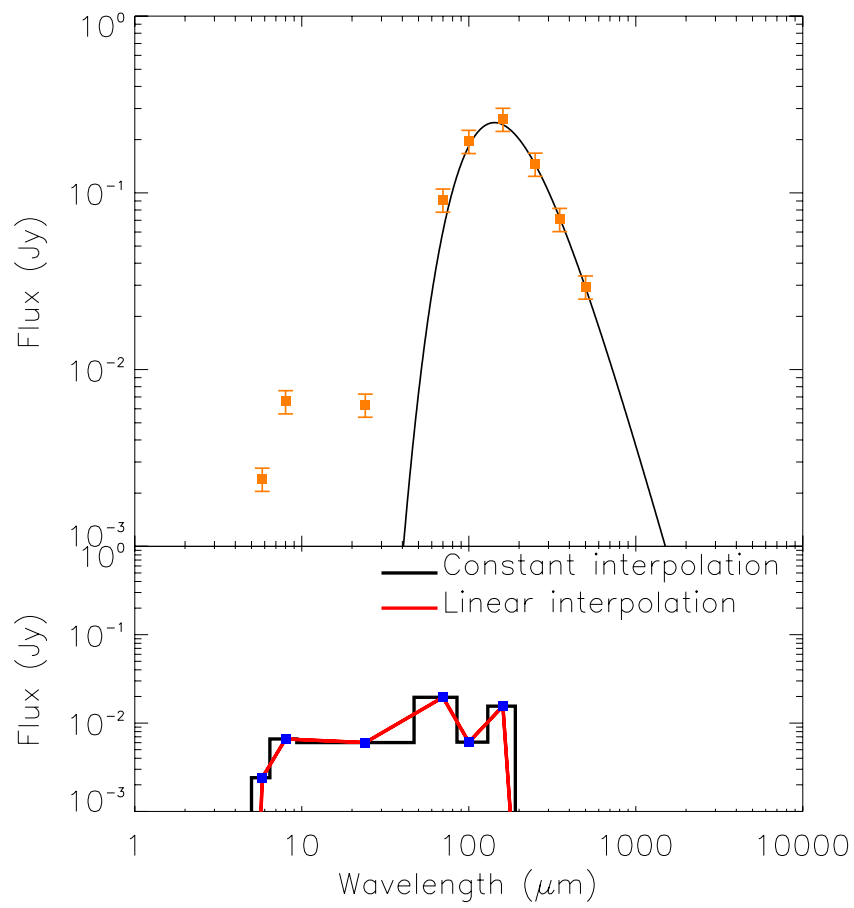

Fig. 5. Two of the techniques adopted to estimate the "warm" dust luminosity. After computing the "cool" dust component (top panel), the residual MIR, FIR, and submm fluxes were calculated (blue points in the bottom panel). These residual fluxes were then integrated using (a) constant values within specified wavelength ranges according to the filter passbands (black lines) and (b) a linear interpolation between successive measurements (red lines). The pixel used for this demonstration is centered at 01:34:14.64, +30:34:31.57.

the galaxy (further than the distances allowed by the 3- $\sigma$ criterium to all available observations), we constrained the model by using the $24,70,250,350$, and $500 \mu$ m fluxes only (i.e., dropping the low signal-to-noise 100 and $160 \mu \mathrm{m}$ measurements in the outer parts of the galaxy). These signal-to-noise considerations allowed $47 \%$ of the galaxy's area to be modeled by using the full dataset (seven wavelengths), while for the remaining $53 \%$ (mostly the outermost parts of the galaxy), the model was constrained by measurements at five wavelengths. We note here that, at that point of the analysis, we made no use of the 5.8 and $8 \mu \mathrm{m}$ fluxes to constrain the model. These observations were instead used later to calculate the "warm" dust component of the SED (see Sect. 4.1). To adjust for the filter bandpasses, the SED was convolved with the filter transmission before entering the $\chi^{2}$ minimization process. On the basis of the $\chi^{2}$ minimization, we derived best-fit temperatures and luminosity densities.

To compute the uncertainty in the derived parameters, we fitted the model to fluxes obtained within the uncertainty range of the observed fluxes. This was done by generating a series of random deviates with a normal distribution (centered on the observed flux; Press et al. 1986). We created 2000 of these mock datasets which, after fitting the SED model, resulted in a set of 2000 values for each fitted parameter. From this distribution, the $90 \%$ confidence intervals were determined and both lower and upper limits were assigned to the best-fit value of each parameter.

The model dependence on the dust emissivity index $\beta$ was first explored by examining the change in $\chi^{2}$ for different $\beta$ values. Figure 3 shows the histogram of the reduced $\chi_{\text {red }}^{2}$ values (the $\chi^{2}$ divided by the number of observed parameters minus the number of the fitted parameters minus one) for $\beta=1,1.3,1.5$, 


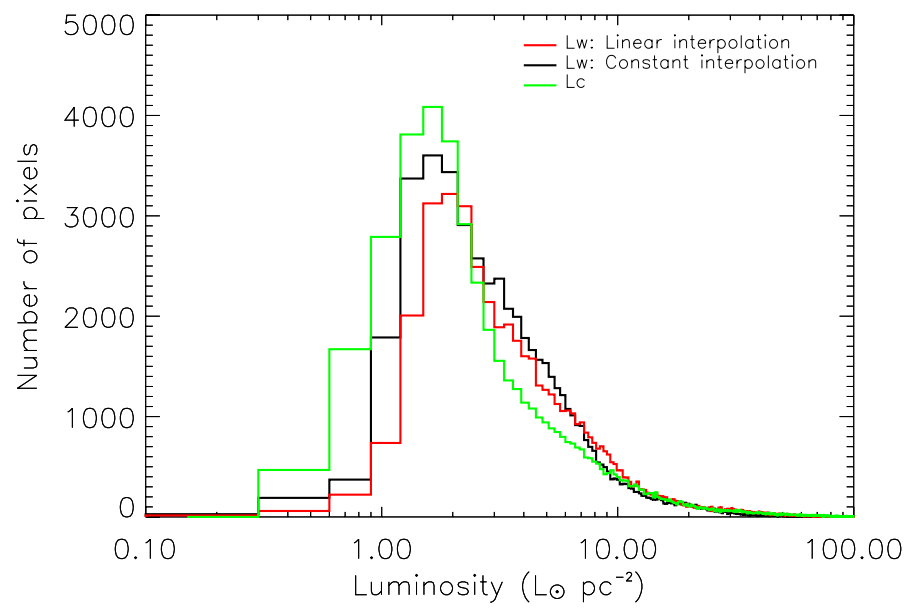

Fig. 6. The histogram of the luminosity densities of M 33 produced by the "cool" dust component (green) and the "warm" dust component (red and black). The "cool" part of the luminosity was derived by fitting the SED with a two-component modified blackbody $(\beta=1.5)$ model. The "warm" part of the luminosity is computed using the residual fluxes of the SED, derived after subtracting the "cool" dust component (see Fig. 5), and by adopting two approximations consisting of integrating the residual SED by a constant interpolation (black line) and a linear interpolation (red line) between the successive fluxes (see the text for more details).

1.7, and 2. From this plot, it is evident that the values close to $\beta=1.5$ account for more points corresponding to the minimum value of $\chi_{\text {red }}^{2}$, which implies that there is a closer match between observations and the model within the error variance of the data. The peak values of the histograms correspond to values of $\chi_{\text {red }}^{2}$ of $\sim 0.17,0.02,0.02,0.06$, and 0.19 for $\beta=1,1.3,1.5,1.7$, and 2 , respectively, with the $\beta=1.5$ distribution being the narrowest one. This value of $\beta$ is in good agreement with the best-fit value found by Kramer et al. (2010) in azimuthally averaged fluxes in elliptical rings. It is also in good agreement with statistical studies of galaxies (e.g., Dunne \& Eales 2001; Yang \& Phillips 2007; Benford \& Staguhn 2008). A comprehensive analysis of the dust emission dependence on $\beta$ (within the framework of the HerM33es Key Project) is presented elsewhere (Tabatabaei et al. 2011).

\subsection{The "warm" dust component of the SED}

The "warm" dust component is a poorly constrained parameter of our model, because the simple model that we use is incapable of accurately describing the heating of the small dust grains emitting at short wavelengths. This is a particularly important caveat of the model when fitting bright parts of the galaxy such as the central region and the cores of the bright HII regions. In these regions, the majority of the SED can be adequately fitted by the "cool" component, leaving only one flux measurement (at $24 \mu \mathrm{m}$ ) to constrain the "warm" part of the SED. It is, nevertheless, the unphysically large values of the "warm" dust temperatures and luminosities as well as the large uncertainties in these parameters that make this model inadequate in accounting for this part of the SED. A more realistic model, taking into account dust emission at shorter, MIR, wavelengths is clearly needed for a more complete determination of the dust properties of the galaxy (Rosolowski et al., in prep.).

Typical examples of single-pixel SEDs are shown in Fig. 4. The upper right panel presents an SED near the peak of the NGC 604 HII region $(01: 34: 34.09,+30: 46: 51.25)$, the lower left

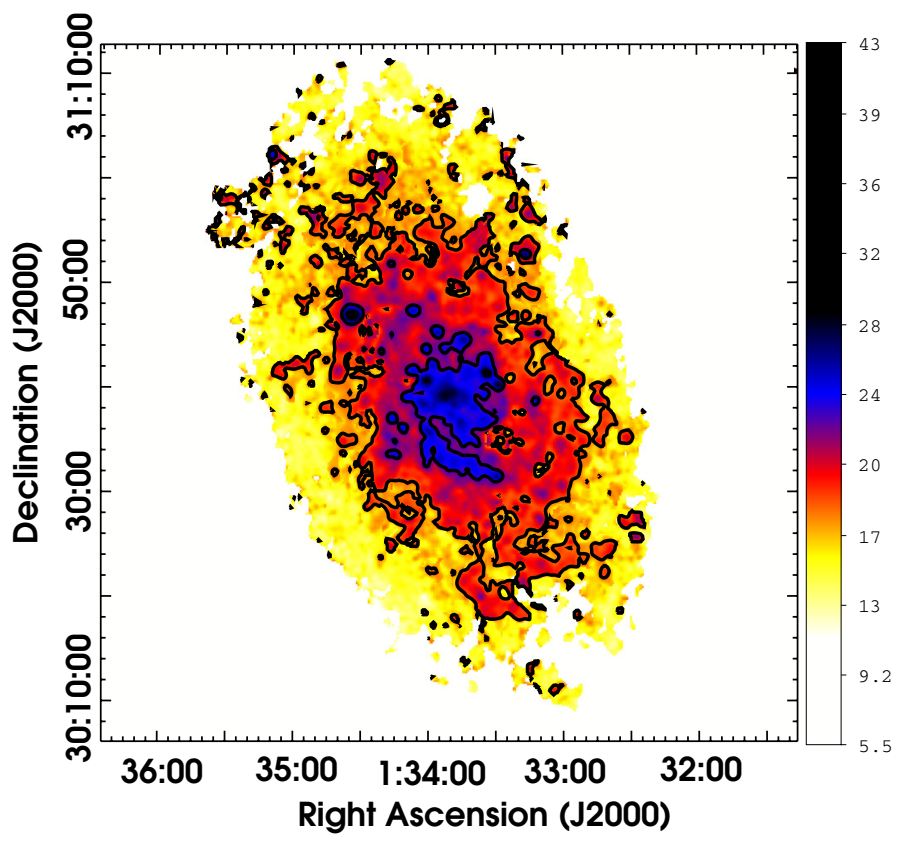

Fig. 7. The "cool" temperature $\left(T_{\mathrm{c}}\right)$ distribution across the galaxy. The two isothermal contours delineate regions with $T_{\mathrm{c}}<18 \mathrm{~K}$ (yellow areas), $18 \mathrm{~K}<T_{\mathrm{c}}<23 \mathrm{~K}$ (red/orange areas), and $T_{\mathrm{c}}>23 \mathrm{~K}$ (blue areas).

panel shows an SED at the center of the galaxy (01:33:45.21, $+30: 38: 41.69)$, and a faint, diffuse part of the outskirts of the galaxy $(01: 32: 42.54,+30: 30: 50.58)$ is presented in the lower right panel. The SED for the last position shows a typical case where only five flux measurements (PACS excluded; see Sect. 4) are used to constrain the model. From these SEDs, the lack of data points shorter than $24 \mu \mathrm{m}$ becomes evident in the brightest parts of the galaxy (NGC 604 and center of the galaxy) with the model failing to simulate the "warm" dust emission giving unphysically high values for the temperature of this component.

To estimate the energy output of M 33 linked to the starforming activities taking place inside the galaxy (the "warm" part of the SED), we computed the luminosity of the residual fluxes, after subtracting the well-defined "cool" component of the SED computed above; see Fig. 5. This was done on a pixelby-pixel basis, by integrating the residual fluxes assuming a constant interpolation between successive wavelengths (see an example in Fig. 5). In particular, we assumed that the 5.8, 8, 24, $70,100,160,250,350$, and $500 \mu \mathrm{m}$ residual fluxes were constant in the wavelength ranges of 5-6.44 $\mu \mathrm{m}, 6.44-9.34 \mu \mathrm{m}$, 9.34-47 $\mu \mathrm{m}, 47-85 \mu \mathrm{m}, 85-130 \mu \mathrm{m}, 130-190 \mu \mathrm{m}, 190-300 \mu \mathrm{m}$, $300-400 \mu \mathrm{m}$, and $400-1000 \mu \mathrm{m}$, respectively (see the residual plot in Fig. 5). The wavelength ranges for the 5.8, 8, 250, 350, and $500 \mu \mathrm{m}$ residual fluxes were dictated by the actual width of the IRAC and SPIRE passbands, with the $500 \mu \mathrm{m}$ range extending up to $1000 \mu \mathrm{m}$. For the MIPS and the PACS measurements, we used the mid-point between successive wavelengths as the wavelength limit. With this method, we computed a "warm" dust luminosity of $7.8 \times 10^{8}+2.1 \times 10^{8} L_{\odot}$ across the entire galaxy. We note here that the tightly constrained "cool" dust component results in a luminosity production of $1.1 \times 10^{9}{ }_{-2.0 \times 10^{8}}^{+3.9 \times 10^{8}} L_{\odot}$.

To test the accuracy of this method, we also computed the "warm" dust luminosity by integrating the residual fluxes but, this time, assuming a linear interpolation for the residual fluxes at the specific wavelengths (Fig. 5). With this method, we computed a "warm" dust luminosity of $1.0 \times 10^{9}+3.0 \times 10^{8} L_{\odot}$. A more detailed comparison, on a pixel by pixel basis, between the two 


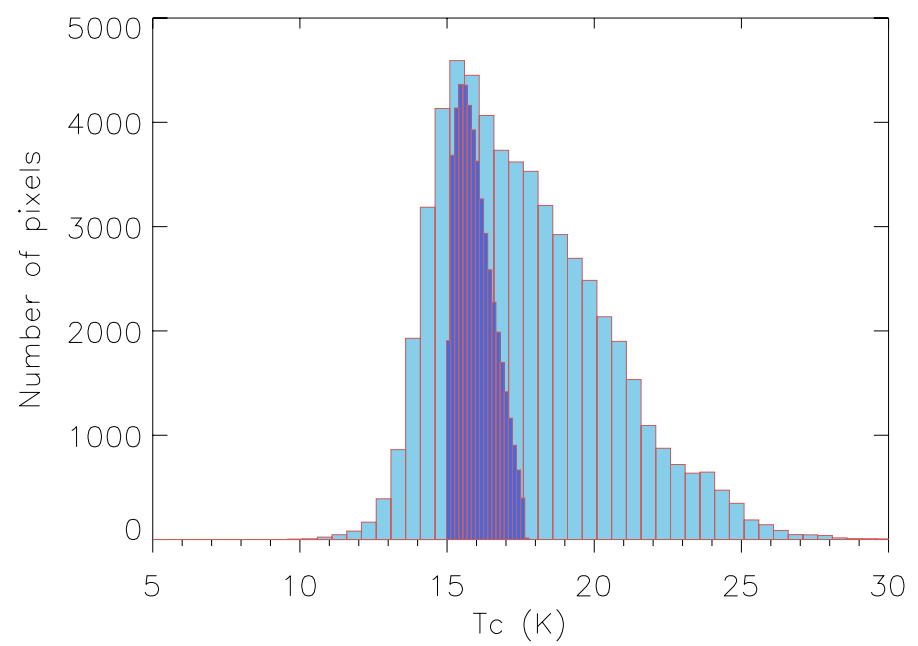

Fig. 8. The "cool" temperature $\left(T_{\mathrm{c}}\right)$ histogram distribution. The values derived for the whole galaxy are shown in the light blue histogram, while the dark blue only accounts for the diffuse disk of the galaxy (see Sect. 6.2).

methods to determine the "warm" dust luminosity, as described above, is given in Fig. 6 with the histograms of the luminosity density (in units of $L_{\odot} \mathrm{pc}^{-2}$ ) presented for the two cases discussed above. For comparison, the histogram of the "cool" luminosity density is also plotted. From these histograms, one can see that the distribution of the luminosity densities using the two methods is very similar with the "constant" interpolation approach giving a slightly broader distribution than the "linear" one.

A further check that we did in order to investigate the accuracy of our method was to compute the luminosities of the two components by using the total fluxes for the galaxy (integrated within an ellipse with a semi-major axis length of $8 \mathrm{kpc}$ ). With this approach, both the "cool" and the "warm" components could be calculated (see Kramer et al. 2010), resulting in a "cool" dust luminosity of $9.3 \times 10_{-2.2 \times 10^{8}}^{+3.7 \times 10^{8}} L_{\odot}$ and a "warm" dust luminosity of $7.4 \times 10^{8}+2.6 \times 10^{8} L_{\odot}$ (see upper left panel in Fig. 4 ).

As a final test, we computed the total luminosity of M 33 following the recipe presented in Boquien et al. (2011). With this approach, the total IR luminosity was computed by fitting the Draine \& Li model to the data (IRAC, MIPS, PACS, SPIRE). As a product of this method, scaling relations were produced between surface brightness measurements at specific wavelengths and the total IR luminosity. For our purposes, we chose to use the $250 \mu \mathrm{m}$ surface brightness map, which is the one with the best signal-to-noise. After calculating the total IR luminosity and subtracting the already estimated "cool" dust luminosity, we found a "warm" luminosity of $8.4 \times 10^{8} L_{\odot}$.

Given the large differences between the methods discussed above, the values for the "warm" dust luminosity are very similar, within the computed uncertainties, giving us great confidence that this part of the luminosity, although estimated with very simple methods, is an accurate first approximation. In our subsequent analysis, we used the luminosity calculations derived with the "constant" interpolation method since with this method the total "warm" luminosity is closer to the values derived by both the Boquien et al. (2011) method and the integrated fluxes method. With this method, the total luminosity of the galaxy (integrated in the $5-1000 \mu \mathrm{m}$ wavelength range) is $1.9 \times 10^{9}{ }_{-4.4 \times 10^{8}}^{+4.0 \times 10^{8}} L_{\odot}$.

A74, page 6 of 11

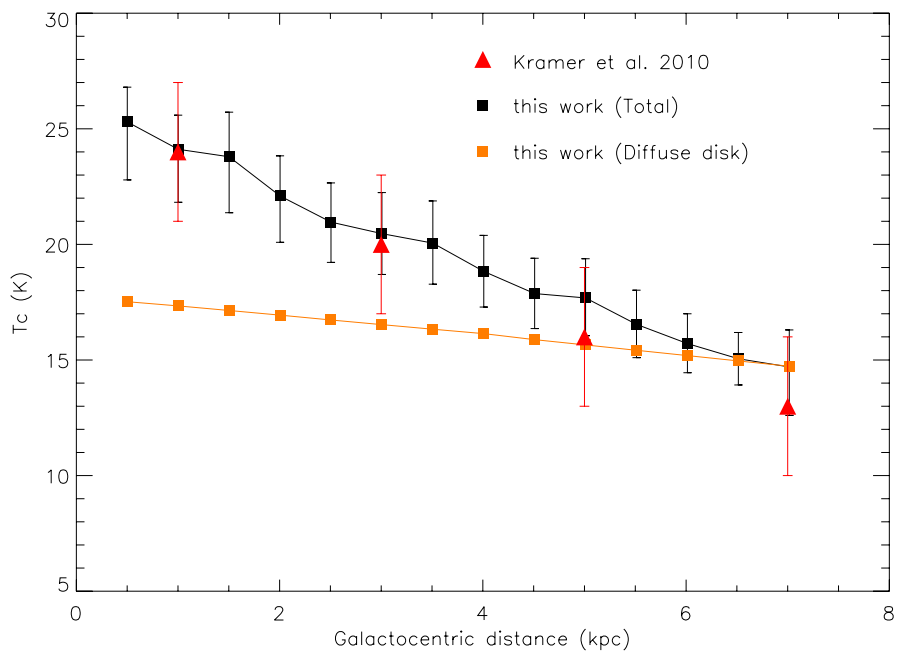

Fig. 9. Azimuthally averaged radial profile of the "cool" temperature for the whole galaxy (black sqares) and the disk only (orange squares; see Sect. 6.2). For comparison, the "cool" dust temperatures for the dust that were calculated in Kramer et al. (2010) for a two-component modified blackbody model with $\beta=1.5$ and the radial anulii $0<R<$ $2 \mathrm{kpc}, 2<R<4 \mathrm{kpc}$, and $4<R<6 \mathrm{kpc}$ are also presented (red triangles).

\section{Results}

Figure 7 shows the "cool" temperature pixel map created by modeling the SED. This dust component is heated to temperatures of between $11 \mathrm{~K}$ and $28 \mathrm{~K}$ with $\sim 15 \mathrm{~K}$ being the most common temperature throughout the galaxy (see Fig. 8). In general, the "cool" temperature is symmetrically distributed throughout M 33 with typical temperatures of $\sim 25 \mathrm{~K}$ close to the center of the galaxy, dropping to $\sim 15 \mathrm{~K}$ close to the outskirts. The spiral structure can not be clearly seen in the "cool" temperature distribution (Fig. 7). The effect of the dimming of the spiral arms with respect to the large radial gradient of the galaxy is also present when looking at the 250/350 and 500/350 color maps (with the SPIRE colors being valuable indicators of the "cool" dust temperature distribution; see Fig. 4 in Boquien et al. 2011; and Fig. 1 in Braine et al. 2010). This is also evident when looking at the azimuthally averaged profile of the temperature (black line in Fig. 9), which undergoes a monotonic decrease from the center to the outskirts with no obvious variations from, e.g., the spiral structure. For comparison, the "cool" dust temperature values calculated by Kramer et al. (2010) but for a range of elliptical annuli are also plotted (red triangles). In general, the values are within the errors in the temperature. The slightly higher temperatures that we calculate are due to the small differences in the calibration of the Herschel data set used in Kramer et al. (2010).

Temperatures of $T<18 \mathrm{~K}$ (yellow areas in Fig. 7) are found in the outer parts of the galaxy, while higher temperatures are found in the inner parts of the galaxy. The highest temperatures ( $T>23 \mathrm{~K}$; innermost contour; blue areas in Fig. 7) are found in individual HII regions and at the center of the galaxy. This picture is also evident when examining individual SEDs similar to the ones presented in Fig. 4 with temperatures in the ranges discussed above for the HII regions (e.g., NGC 604), the center of the galaxy, and the diffuse outskirt regions of the galaxy. In these cases, the best-fit temperatures and the $90 \%$ confidence limits obtained with this model are $31.5_{-5.1}^{+1.6} \mathrm{~K}$ for the NGC 604 region, $25.4_{-1.8}^{+1.0} \mathrm{~K}$ for the central region of the galaxy, and $15.7_{-3.0}^{+4.7} \mathrm{~K}$ in the outer, diffuse parts of the galaxy. 


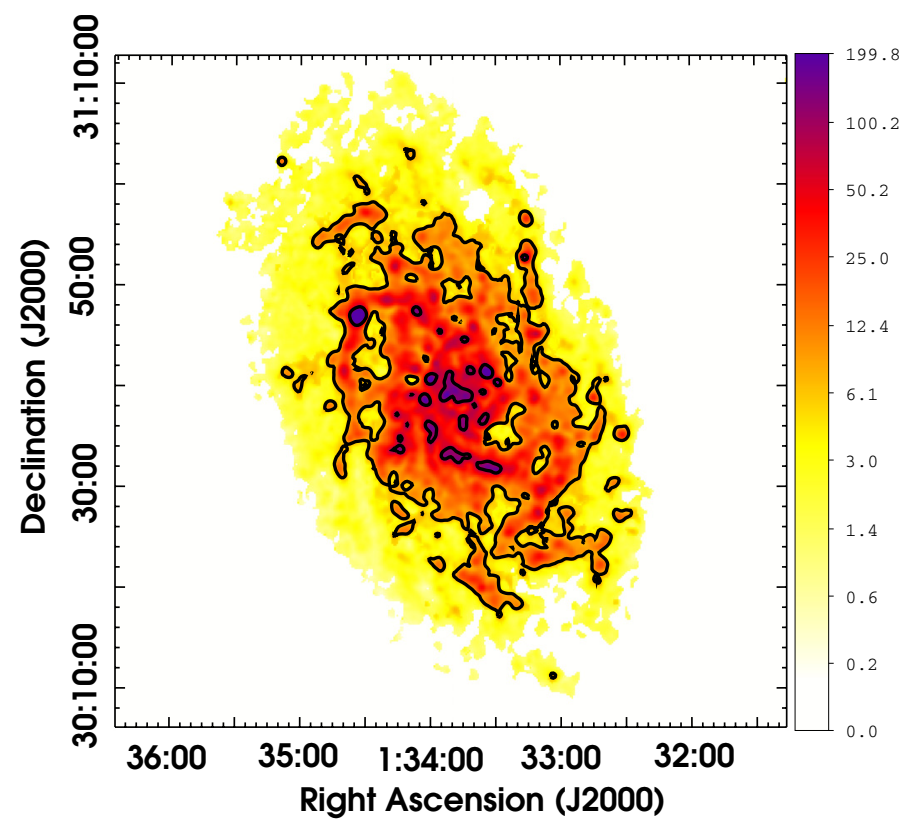

Fig. 10. The luminosity map of M 33 produced by the "cool" dust grains. Luminosities fainter than $7 L_{\odot} \mathrm{pc}^{-2}$ (yellow areas) are found in the outer diffuse parts of the galaxy, while higher luminosities trace the spiral arms (orange/red areas). The highest luminosity densities ( $>90 L_{\odot} \mathrm{pc}^{-2}$; innermost contours; blue areas) are produced in the bright HII regions and the center of the galaxy.

These are typical temperatures for the various regions throughout the galaxy, which also provide an estimate of the accuracy of the fitted parameters. Given the simplicity of our SED model, especially at MIR wavelengths (those shorter than $\sim 70 \mu \mathrm{m}$ ), where significant contribution to the emission from stochastically heated dust grains is expected, we investigated the effects of omitting the $70 \mu \mathrm{m}$ fluxes from the analysis. In this way, the "cool" dust component was constrained by observations at wavelengths longer than $100 \mu \mathrm{m}$. We found that the "cool" dust temperature varies only slightly (by less than $2 \%$ ), which is well within the estimated uncertainties. This gives us sufficient confidence in our "cool" dust component calculation.

For the "warm" dust component, we do not present a temperature map since, as already stressed before, the temperature of this component derived from the two modified-blackbody fit, is highly uncertain in many regions throughout the galaxy (especially the bright ones; see the upper right and lower left panels in Fig. 4). It is worth mentioning however that, when considering the galaxy as a whole, the "warm" dust temperature can be calculated, and is $55.0_{-3.7}^{+2.5} \mathrm{~K}$ (in accordance with previous studies, e.g., Kramer et al. 2010) while for a typical, low brightness region (similar to the one in the lower right panel of Fig. 4), the temperature is $51.0_{-2.3}^{+2.9} \mathrm{~K}$.

The "cool" dust luminosity (Figs. 6 and 10) varies between $\sim 0.3 L_{\odot} \mathrm{pc}^{-2}$ and $\sim 800 L_{\odot} \mathrm{pc}^{-2}$ with a value of $\sim 1.7 L_{\odot} \mathrm{pc}^{-2}$ accounting for most parts of the galaxy. Luminosities fainter than $7 L_{\odot} \mathrm{pc}^{-2}$ (yellow areas in Fig. 10) were detected for the diffuse parts of the outermost regions of the galaxy, while higher luminosities (orange/red areas in Fig. 10) are emitted from the spiral arms. The highest luminosity densities $\left(L>90 L_{\odot} \mathrm{pc}^{-2}\right.$; innermost contours (blue areas) in Fig. 10) are produced by the HII regions and the center of the galaxy. This is also evident when examining the individual SEDs for different regions of the galaxy (Fig. 4), resulting in a "cool" dust luminosity of

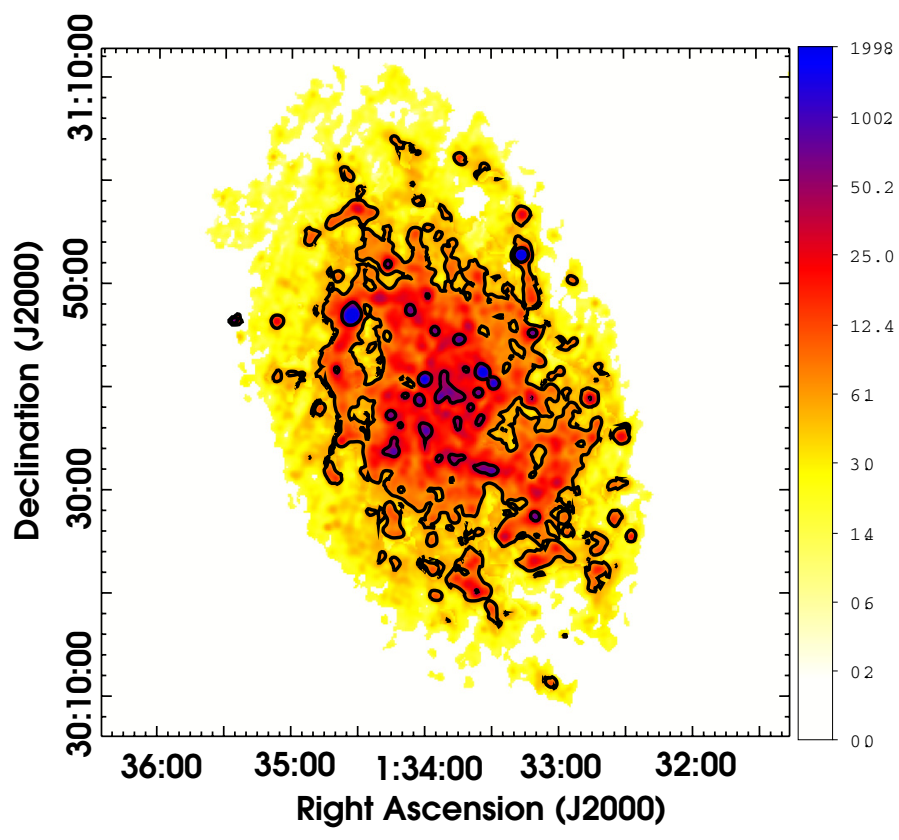

Fig. 11. The luminosity map of M 33 produced by the "warm" dust grains. Luminosities fainter than $6 L_{\odot} \mathrm{pc}^{-2}$ (yellow areas) are found in the outer diffuse parts of the galaxy, while higher luminosities trace the spiral arms (orange/red regions). The highest luminosity densities ( $>40 L_{\odot} \mathrm{pc}^{-2}$; innermost contours; blue areas) are produced in the bright HII regions and the center of the galaxy. The "constant" interpolation method was used to estimate the "warm" dust luminosities.

$819_{-415}^{+84} L_{\odot} \mathrm{pc}^{-2}$ for NGC 604 , one of $114_{-24}^{+12} L_{\odot} \mathrm{pc}^{-2}$ for the center, and $2.2_{-0.95}^{+2.4} L_{\odot} \mathrm{pc}^{-2}$ for the outer parts of the galaxy.

The "warm" dust luminosity (Figs. 6 and 11) varies between $\sim 0.3 L_{\odot} \mathrm{pc}^{-2}$ and $\sim 400 L_{\odot} \mathrm{pc}^{-2}$ with a value of $\sim 1.6 L_{\odot} \mathrm{pc}^{-2}$ accounting for most parts of the galaxy. Luminosities less than $7 L_{\odot} \mathrm{pc}^{-2}$ (yellow areas in Fig. 11 ) are produced in the diffuse parts of the galaxy while higher luminosities are emitted from the spiral arms (orange/red areas in Fig. 11). The highest luminosity densities $\left(L>40 L_{\odot} \mathrm{pc}^{-2}\right.$; innermost contours (blue areas) in Fig. 11) are produced by the HII regions and the center of the galaxy. This map was computed by applying the "constant" interpolation method for estimating the "warm" dust luminosity (see Sect. 4.1). As in the case of the "cool" dust luminosity (discussed above) individual SEDs correspond to luminosities, produced by the "warm" dust material, of $404_{-67}^{+380} L_{\odot} \mathrm{pc}^{-2}$ for NGC 604, $42_{-27}^{+18} L_{\odot} \mathrm{pc}^{-2}$ for the center of M33, and $2.8_{-1.97}^{+0.6} L_{\odot} \mathrm{pc}^{-2}$ for the outer parts of the galaxy.

\section{Discussion}

\subsection{The "warm" and the "cool" dust distributions}

Our comparison of the "cool" and the "warm" luminosity maps in Figs. 10 and 11 indicates that the morphologies of the two dust components are very similar with the spiral structure clearly being seen in both cases and with local enhancements being found in the areas where the HII regions reside. This similarity becomes more striking when looking at the azimuthally averaged radial profiles (upper panel in Fig. 12) of the luminosity densities of the two components (green and blue lines for the "cool" and the "warm" components) and their ratio $\left(L_{\mathrm{c}} / L_{\mathrm{w}}\right.$; red line). From these profiles, it is evident that, given the uncertainties in the computed luminosities, the "cool" dust luminosity is the dominant source of luminosity in the central parts of the galaxy (with 


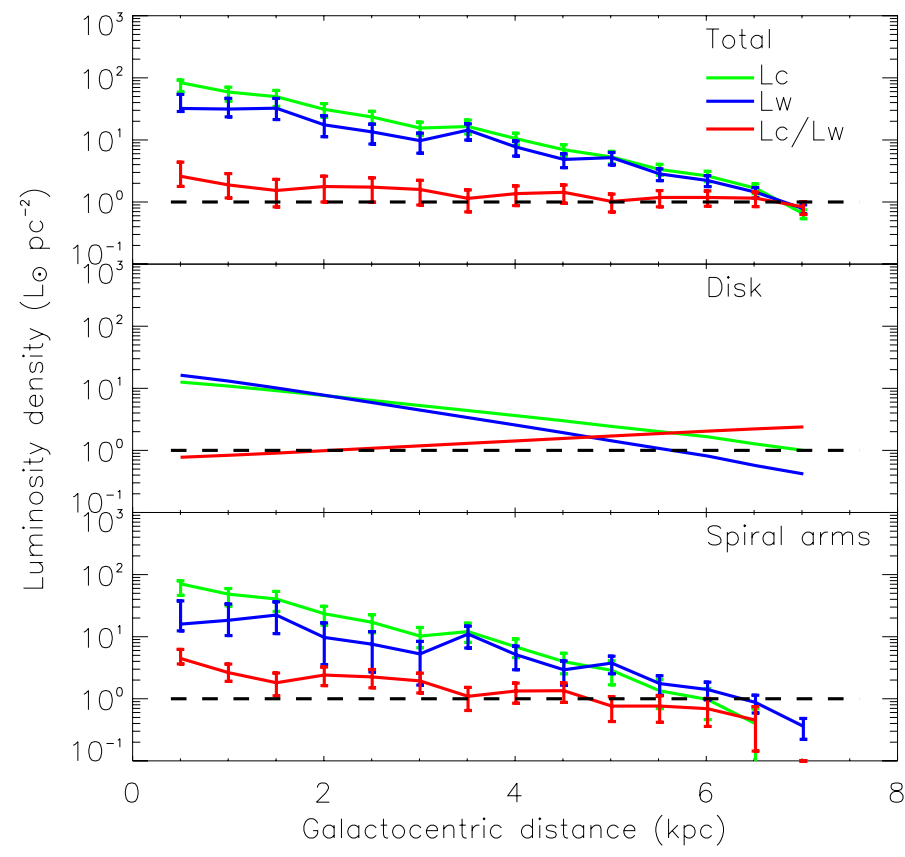

Fig. 12. The azimuthally averaged profiles of the "cool" and the "warm" dust luminosities (green and blue lines) in units of $L_{\odot} \mathrm{pc}^{-2}$. The coolto-warm luminosity ratio is also plotted (red line). The luminosities are calculated for the whole galaxy (upper panel) and the disk of the galaxy only (middle panel; see Sect. 6.2). The residuals between the two profiles give the radial distribution of the luminosity produced by the spiral arm network (bottom panel).

the "cool" luminosity being about three times higher than the "warm" luminosity), while the ratio gets rather flat and close to unity beyond a $\sim 3 \mathrm{kpc}$ radius. This narrow range of the ratio (close to unity) of the two luminosities suggests that both dust components (the "cool" dust heated at $\sim 15 \mathrm{~K}$ and the "warm" dust heated at $\sim 55 \mathrm{~K}$ ) are able to produce about the same luminosities throughout the galaxy.

Approximating the azimuthally averaged radial profiles of the two luminosities with exponential functions, we find that they decay with scalelengths of $2.59 \pm 0.04 \mathrm{kpc}$ for the "cool" dust, and $1.77 \pm 0.02 \mathrm{kpc}$ for the "warm" dust. Comparing with the scalelengths that we have derived for individual wavelengths (Sect. 3 and this section), we can see that the "warm" dust luminosity is very similar to the values derived for the MIR/FIR wavelengths, while the "cool" dust luminosity has a scalelength similar to the $250-500 \mu \mathrm{m}$ submm wavelengths. This directly implies that the "cool" dust grains (which contribute mostly to the "cool" part of the luminosity of the galaxy) emit in the submm wavelengths, while the "warm" dust grains emit in the MIR/FIR wavelengths forming the "warm" part of the luminosity of the galaxy.

\subsection{Decomposing M33 into a disk and a spiral arm network}

The dust distribution inside a galaxy can be quite inhomogeneous. When considering the morphology on a large scale, the dust distribution can be approximated by a superposition of a diffuse disk and a spiral arm network (see e.g. Xilouris et al. 1999; Misiriotis et al. 2000; Meijerink et al. 2005). As a first order approximation, the diffuse disk can be accurately described by a three-dimensional disk decreasing exponentially in both the radial and the vertical directions

$$
\eta(R, z)=\eta_{0} \exp \left(-\frac{R}{h_{\mathrm{d}}}-\frac{|z|}{z_{\mathrm{d}}}\right),
$$

where $\eta_{0}$ is the dust volume density at the nucleus of the galaxy and $h_{\mathrm{d}}$ and $z_{\mathrm{d}}$ are the scalelength and scaleheight of the dust, respectively (see Xilouris et al. 1999). We used this simple model to delineate between the two main dust components (i.e. the dust that is diffusely distributed in a disk throughout the galaxy revealing itself in the inter-arm regions of the galaxy and the dust forming the spiral arms).

Determining the scaleheight of the dust is non-trivial since, unlike for edge-on galaxies where the vertical extent of the dust can be measured, in less-inclined galaxies, such as M33, this parameter cannot be easily determined. We note however that in our present study, where we aim to calculate the relative fraction of the fluxes and luminosities emitted by the diffuse and the spiral arm network, the choice of the scaleheight is not a crucial issue. This is because the total luminosity of the disk is independent of $z_{\mathrm{d}}$ (a larger value of $z_{\mathrm{d}}$ (thicker disk) would require a smaller value of $\eta_{0}$ (fainter disk) in order to fit the data, which, in the end, would also result in the same luminosity; see Xilouris et al. 1997). For the purposes of this study, we used a scaleheight of $z_{\mathrm{d}}=0.2 \mathrm{kpc}$. This is a typical scaleheight for the diffuse dust in a galaxy derived from modeling and observations of edge-on galaxies (Xilouris et al. 1999; Bianchi \& Xilouris 2011). In particular, for M 33, it is close to the value derived by Combes et al. (2012) using a power-spectrum analysis.

For the scalelength, we used the values determined by fitting the azimuthally averaged profiles (see Sect. 3, Fig. 2). Since we also wished to investigate the origin of the emission at MIR wavelengths, we performed the analysis for the scalelength determination (described in Sect. 3) in the MIPS-24 $\mu \mathrm{m}$ and $70 \mu \mathrm{m}$ data. We found scalelength values of $1.87 \pm 0.09$ and $1.90 \pm 0.05 \mathrm{kpc}$, respectively, for the $24 \mu \mathrm{m}$ and $70 \mu \mathrm{m}$ emission.

With the scalelengths and scaleheights of the disk fixed, our three-dimensional model was then inclined by 56 degrees (the inclination angle of M33) and the intensities of the model integrated along the line of sight were fitted to the galaxy maps on a pixel-by-pixel basis. We used only the fluxes in the outermost and the inter-arm regions of the galaxy (the latter areas consisting of the minimum values in-between the obvious spiral structure) to constrain the emissivity at the galactic center $\left(\eta_{0}\right)$. We did this because we only wished to fit the diffuse part of the galaxy and disregard both the spiral arms and the more complex structures superimposed on the diffuse disk. This fit was done by minimizing the $\chi^{2}$ values in a similar procedure with what is described in Sect. 4.

An example of this analysis is presented in Fig. 13, which illustrates the $250 \mu \mathrm{m}$ map decomposition. In this figure, the black contours show the axisymmetric exponential disk that is fitted to the data (Eq. (2)), while the red contours show the spiral arm network determined after subtracting the map of the diffuse disk from the observed image. This decomposition can be seen in more detail in Fig. 14, where we present the radial profiles of the disk fitted to the $250 \mu \mathrm{m}$ data (dotted lines) along with the observed major- and minor-axis profiles (left and right panel respectively). From these profiles, it is clear that the spiral arms are superimposed on the disk, allowing a clear separation of the two components.

Using the available Spitzer and Herschel maps of M33, we found that the flux that is emitted from the disk accounts for 21 , $29,38,42,48,52$, and $57 \%$ of the total flux at the wavelengths 
Table 1. "Warm" and "cool" dust luminosities for the total galaxy, the disk of the galaxy and the spiral arms of the galaxy.

\begin{tabular}{l}
\hline \hline Total galaxy \\
\hline$L_{\text {total }}=1.9 \times 10^{9} L_{\odot}$ \\
$L_{\mathrm{w}}=7.8 \times 10^{8} L_{\odot}\left(41 \% L_{\text {total }}\right)$ \\
$L_{\mathrm{c}}=1.1 \times 10^{9} L_{\odot}\left(59 \% L_{\text {total }}\right)$ \\
\hline Disk \\
\hline$L_{\text {disk }}=5.7 \times 10^{8} L_{\odot}\left(30 \% L_{\text {total }}\right)$ \\
$L_{\text {disk }}^{\mathrm{w}}=2.5 \times 10^{8} L_{\odot}\left(32 \% L_{\mathrm{w}}, 44 \% L_{\text {disk }}\right)$ \\
$L_{\text {disk }}^{\mathrm{c}}=3.2 \times 10^{8} L_{\odot}\left(29 \% L_{\mathrm{c}}, 56 \% L_{\text {disk }}\right)$ \\
\hline Spiral arms \\
\hline$L_{\text {spiral }}=1.3 \times 10^{9} L_{\odot}\left(70 \% L_{\text {total }}\right)$ \\
$L_{\text {spiral }}^{\mathrm{w}}=5.3 \times 10^{8} L_{\odot}\left(68 \% L_{\mathrm{w}}, 40 \% L_{\text {spiral }}\right)$ \\
$L_{\text {spiral }}^{\mathrm{c}}=7.8 \times 10^{8} L_{\odot}\left(71 \% L_{\mathrm{c}}, 60 \% L_{\text {spiral }}\right)$ \\
\hline
\end{tabular}

$24,70,100,160,250,350$, and $500 \mu \mathrm{m}$, respectively. We can see that at MIR wavelengths, it is the spiral structure that is the dominant source of emission, while, at around $\sim 250 \mu \mathrm{m}$ the emission comes equally from the disk and the spiral arms. In contrast, the dominant source of the submm emission is the disk of the galaxy.

After calculating the emission that is produced by the diffuse dust disk in all available bands, we were then able to calculate the temperature and the luminosity of this component separately. We did so by applying the SED modeling that we had previously performed for the whole galaxy (Sect. 4) but now for this component only.

For the "cool" temperature in the disk, we found a very narrow distribution (compared to the galaxy as a whole) with values ranging between $15 \mathrm{~K}$ and $18 \mathrm{~K}$. This is indicated in Fig. 8 by the dark blue distribution being that of the diffuse disk. Given a typical $3 \mathrm{~K}$ uncertainty in the temperature distribution, the disk temperatures occupy the lower end of the temperature distribution for the whole galaxy (light blue), leaving the higher temperatures $(>18 \mathrm{~K})$ accounting for the rest of the galaxy (spiral arms and HII regions). The narrow range of temperatures is also seen in Fig. 9, with temperatures of $\sim 18 \mathrm{~K}$ occuring at the center of the galaxy dropping to $\sim 15 \mathrm{~K}$ at the edges. A similarly narrow range of temperatures for the diffuse dust in the disk of the Milky Way was derived based on COBE observations. Temperatures for the diffuse cirrus component ranging from $17 \mathrm{~K}$ to $21 \mathrm{~K}$ were found using FIR color maps (e.g. Reach et al. 1995; Schlegel et al. 1998), while a $15 \mathrm{~K}$ to $19 \mathrm{~K}$ range was computed for the disk of the Milky Way via radiative transfer modeling (Misiriotis et al. 2006). Similar findings were derived using Herschel observations of the Galactic plane indicating that the warmest dust is located in the spiral arms (Bernard et al. 2010). The narrow range of the temperatures that we measured for the disk of M33 is consistent with this picture, with the disk primarily hosting the cirrus dust component, which is in turn heated by the diffuse interstellar radiation field. On the other hand, the dust in the spiral arms is embedded in more dense environments, and is mainly heated by the local ultraviolet radiation of the young stars, leading to a wider range of temperatures. We note however that a more detailed analysis, taking into account both the exact radiation field heating the dust as well as the dust composition and density in various places throughout the galaxy, is clearly needed for a proper characterization of the properties of the interstellar dust in different environments.

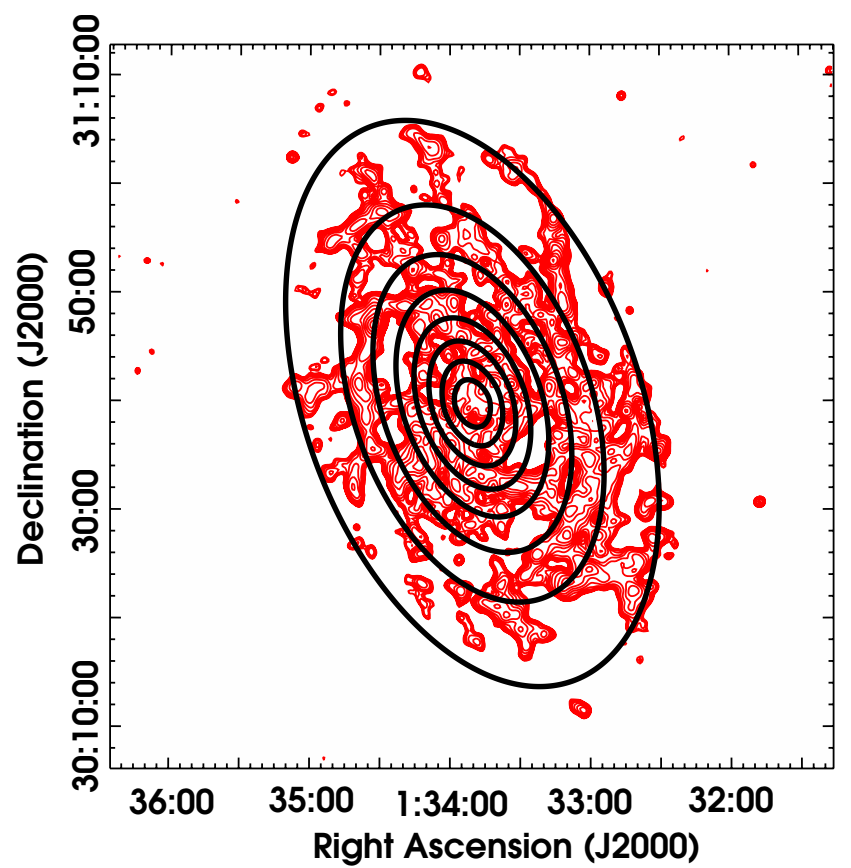

Fig. 13. The $250 \mu \mathrm{m}$ image of M 33 decomposed into a diffuse axisymmetric disk (black contours) and a spiral structure network (red contours; see the text for more details).
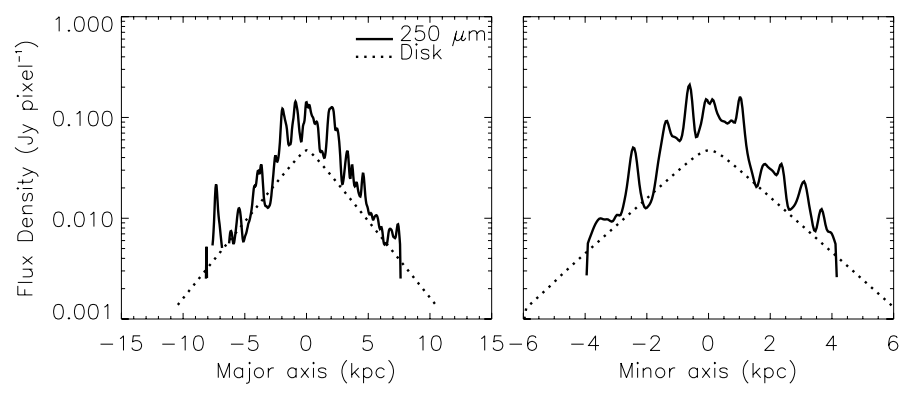

Fig. 14. The $250 \mu \mathrm{m}$ profile of M 33 along both the major axis (left) and the minor axis (right). In both cases, the solid line corresponds to the observed emission, while the dotted line is the exponential disk fitted to the data.

Concerning the luminosity, we find that $5.7 \times 10^{8} L_{\odot}(30 \%$ of the total luminosity) is produced by the dust in the disk. This indicates that the dominant source of the luminosity of the galaxy at IR/submm wavelengths is the spiral arm network. Furthermore, considering the "warm" and the "cool" components separately we computed that the dust that resides in the disk and is "warm" emits a luminosity of $2.5 \times 10^{8} L_{\odot}$, which is $32 \%$ of the total "warm" dust luminosity, while the "cool" dust material produces $3.2 \times 10^{8} L_{\odot}$, which is $29 \%$ of the total "cool" dust luminosity. All the above results, as well as the values derived for the spiral arms, are summarized in Table 1.

The above results indicate that the luminosities of the "warm" and the "cool" dust appear to originate mainly in the spiral arm network, which produces $71 \%$ of the luminosity of the "cool" and $68 \%$ of the luminosity of the "warm" dust of the galaxy. Furthermore, $41 \%$ of the total luminosity of the galaxy is emitted by "warm" grains, while the remaining $59 \%$ is emitted by "cool" dust grains. These percentages are about the same when separating the disk from the spiral arms. For example, we see that inside the disk $44 \%$ of the luminosity is emitted by the "warm" dust, whereas $56 \%$ is emitted by the "cool" 
dust; similarly for the spiral arms, $40 \%$ comes from the "warm" and $60 \%$ from the "cool" dust.

It is also interesting to investigate the relative distributions of the "warm" and "cool" luminosities that reside inside the disk and the spiral arms as a function of galactocentric radius. The decomposition of the azimuthally averaged radial profiles is shown in Fig. 12, with the disk profiles presented in the middle panel and the spiral arm profiles in the bottom panel. The disk profiles (middle panel) are derived by fitting the two modified-blackbody model to the smooth disk maps at each available wavelength. These maps are derived by fitting the three-dimensional model (Eq. (2)) to the observations as described earlier. From these profiles, it is evident that the "cool" dust in the disk of the galaxy becomes the dominant component in the outer parts of the galaxy (beyond $\sim 3 \mathrm{kpc}$ ), while the "cool" dust is the main source of luminosity production within the inner $\sim 3 \mathrm{kpc}$ inside the spiral arm network.

\section{Conclusions}

By modeling the SED of the Local Group galaxy M 33 and exploiting the unique wavelength coverage and sensitivity of the Herschel 100, 160, 250, 350, and $500 \mu \mathrm{m}$ observations, as well as additional Spitzer-IRAC $5.8 \mu \mathrm{m}$ and $8 \mu \mathrm{m}$ and Spitzer-MIPS $24 \mu \mathrm{m}$ and $70 \mu \mathrm{m}$ data, we have derived the following conclusions about the dust emission of the galaxy:

- The submm emission from M33 is distributed more evenly (scalelength of $3.04 \mathrm{kpc}$ at $500 \mu \mathrm{m}$ ) than the far-infrared emission (scalelength of $1.84 \mathrm{kpc}$ at $100 \mu \mathrm{m}$ ).

- On average, the emission by the dust can be adequately modeled by a superposition of two modified blackbodies with $\beta=1.5$.

- The temperature of the "cool" grains ranges between $11 \mathrm{~K}$ and $28 \mathrm{~K}$, with a most common value of $\sim 15 \mathrm{~K}$ across the galaxy.

- The total luminosity of the galaxy (integrated from 5 to $1000 \mu \mathrm{m})$ is $1.9 \times 10^{9}{ }_{-4.4 \times 10^{8}} L_{\odot}$, with the luminosity of the "cool" dust component accounting for $59 \%$ of the total luminosity.

- The scalelength of the "cool" ("warm") luminosity distribution is very similar to that of the submm (MIR/FIR) emission. This similarity directly reflects the wavelengths that contribute the most to each of the two luminosity components.

- Decomposing the galaxy into two components (namely, a diffuse disk and a spiral-arm network), we have found that the emission in the disk accounts for $\sim 21 \%$ of the emission at the MIR wavelengths $(24 \mu \mathrm{m})$, while it gradually rises to $\sim 57 \%$ at submm wavelengths $(500 \mu \mathrm{m})$.

- The "cool" dust material in the disk of the galaxy, presumably accounting for the cirrus dust component of the galaxy, is heated to temperatures of between $\sim 15 \mathrm{~K}$ at the edges of the galaxy and $\sim 18 \mathrm{~K}$ at the center.

- The bulk of the luminosity is emitted by the spiral arm network $(\sim 70 \%)$, and this percentage stays roughly the same when considering the "warm" and the "cool" dust luminosities separately.

- About $\sim 40 \%$ of the total luminosity throughout the galaxy (and separately within the disk and the spiral arms) is emitted by the "warm" dust grains, with the remaining $~ 60 \%$ being emitted by the "cool" dust.
- The ratio of the "cool" to "warm" dust luminosity is close to unity throughout most of the the galaxy but slightly higher within the central $\sim 3 \mathrm{kpc}$ of the galaxy.

- The "cool" dust within the disk becomes the dominant source of the infrared emission in the outer parts of the galaxy (beyond $\sim 3 \mathrm{kpc}$ ).

- In contrast, the "cool" dust within the spiral arms become dominant in the inner parts of the galaxy (within $\sim 3 \mathrm{kpc}$ ).

Acknowledgements. We are grateful to Marc Sauvage, George Bendo, Pierre Chanial, Michael Pohlen, and Richard Tuffs for their help in completing the data reduction. We also thank the anonymous referee for providing useful comments that helped to improve the paper. F.S.T. acknowledges support by the DFG via the grant TA 801/1-1.

\section{References}

Alton, P. B., Trewhella, M., Davies, J. I., et al. 1998, A\&A, 335, 807 Aniano, G. J., Draine, B. T., Gordon, K. D., \& Sandstrom, K. 2011, PASP, 123, 1218

Bendo, G. J., Wilson, C. D., Pohlen, M., et al. 2010, A\&A, 518, L65

Bendo, G. J., Boselli, A., Dariush, A., et al. 2012, MNRAS, 419, 1833

Benford, D. J., \& Staguhn, J. G. 2008, ASPC, 381, 132

Bernard, J.-Ph., Paradis, D., Marshall, D. J., et al. 2010, A\&A, 518, L88

Bevington, P., \& Robinson, D. 1992, Data reduction and error analysis for the physical sciences (McGraw-Hill, Inc.)

Bianchi, S., \& Xilouris, E. M. 2011, A\&A, 531, A11

Binney J., \& Tremaine S. 1987, Galactic Dynamics (Princeton: Princeton Univ. Press)

Block, D. L., Elmegreen, B. G., \& Wainscoat, R. J. 1996, Nature, 381, 674

Block, D. L., Combes, F., Puerari, I., et al. 2007, A\&A, 471, 467

Boquien, M., Calzetti, D., Kramer, C., et al. 2010, A\&A, 518, L70

Boquien, M., Calzetti, D., Combes, F., et al. 2011, AJ, 142, 111

Braine, J., Gratier, P., Kramer, C., et al. 2010, A\&A, 518, L69

Combes, F., Boquien, M., Kramer, C., et al. 2012, A\&A, 539, A67

Davies, J. I., Alton, P., Trewhella, M., Evans, R., \& Bianchi, S. 1999, MNRAS, 304,495

Devereux, N. A., \& Young, J. S. 1990, ApJ, 359, 42

Dunne, L., \& Eales, S. A. 2001, MNRAS, 327, 697

Freedman, W. L., Wilson, C. D., \& Madore, B. F. 1991, ApJ, 372, 455

Gerola, H., \& Seiden, P. E. 1978, ApJ, 223, 129

Gordon, K. D., Bailin, J., Engelbracht, C. W., et al. 2006, ApJ, 638, 87

Gratier, P., Braine, J., Rodriguez-Fernandez, N. J., et al. 2010, A\&A, 522, A3

Gratier, P., Braine, J., Rodriguez-Fernandez, N. J., et al. 2012, A\&A, 542, A108

Griffin, M., Abergel, A., Abreu, A., et al. 2010, A\&A, 518, L3

Haas M., Lemke, D., Stickel, M., et al. 1998, A\&A, 338, 33

Hippelein, H., Haas, M., Tuffs, R. J., et al. 2003, A\&A, 407, 137

Hoopes, C. G., \& Walterbos, R. A. M. 2000, ApJ, 541, 597

Kramer, C., Richer, J., Mookerjea, B., Alves, J., \& Lada, C. 2003, A\&A, 399, 1073

Kramer, C., Buchbender, C., Xilouris, E. M., et al. 2010, A\&A, 518, L67

Krüegel, E. 2003, The physics of interstellar dust, ed. E. Krüegel

Krüegel, E., \& Siebenmorgen, R. 1994, A\&A, 288, 929

Meijerink, R., Tilanus, R. P. J., Dullemond, C. P., Israel, F. P., \& van der Werf, P. P. 2005, A\&A, 430, 427

Misiriotis, A., Kylafis, N. D., Papamastorakis, J., \& Xilouris, E. M. 2000, A\&A, 353,117

Ott, D. 2010, ASP Conf. Ser., 434, 139

Paturel, G., Petit, C., Prugniel. P., et al. 2003, A\&A, 412, 45

Pilbratt, G. L., Riedinger, J. R., Passvogel, T., et al. 2010, A\&A, 518, L1

Poglitsch, A., Waelkens, C., Geis, N., et al. 2010, A\&A, 518, L2

Press, W. H., Flannery, B. P., Tenkolsky, S. A., \& Vetterling, W. T. 1986 Numerical Recipes (Cambridge: Cambridge Univ. Press)

Reach, W. T., Dwek, E., Fixsen, D. J., et al. 1995, ApJ, 451, 188

Regan, M. W., \& Vogel, S. N. 1994, ApJ, 434, 536

Roussel, H. 2012, A\&A, submitted

Schlegel, D. J., Finkbeiner, D. P., \& Davis, M. 1998, ApJ, 500, 525

Tabatabaei, F. S., Beck, R., Krause, M., et al. 2007a, A\&A, 466, 509

Tabatabaei, F. S., Beck, R., Krügel, E., et al. 2007b, A\&A, 475, 133

Tabatabaei, F. S., Braine, J., Kramer, C., et al. 2011, Proc. IAU Symp. 284, eds. R. J. Tiffs, \& C. C. Popescu

Verley, S., Hunt, L. K., Corbelli, E., \& Giovanardi, C. 2007, A\&A, 476, 1161

Verley, S., Corbelli, E., Giovanardi, C., \& Hunt, L. K. 2009, A\&A, 493, 453

Verley, S., Relaño, M., Kramer, C., et al. 2010, A\&A, 518, L68 
Xilouris, E. M., Byun, Y. I., Kylafis, N. D., Paleologou, E. V., \& Papamastorakis, J. 1999, A\&A, 344, 868

Yang, M., \& Phillips, T. 2007, ApJ, 662, 284

Young, J. S., Allen, L., Kenney, J. D. P., Lesser, A., \& Rownd, B. 1996, AJ, 112, 1903

1 Institute for Astronomy, Astrophysics, Space Applications \& Remote Sensing, National Observatory of Athens, P. Penteli, 15236 Athens, Greece

e-mail: xilouris@noa.gr

2 Max-Planck-Institut für Astronomie, Königstuhl 17, 69117 Heidelberg, Germany

3 Laboratoire d'Astrophysique de Marseille - LAM, Université d'Aix-Marseille \& CNRS, UMR7326, 38 rue F. Joliot-Curie, 13388 Marseille Cedex 13, France

4 Instituto Radioastronomia Milimetrica (IRAM), Av. Divina Pastora 7, Nucleo Central, 18012 Granada, Spain

5 Argelander Institut fr Astronomie, Auf dem Hügel 71, 53121 Bonn, Germany

6 Laboratoire d'Astrophysique de Bordeaux, Université Bordeaux 1, Observatoire de Bordeaux, OASU, UMR 5804, CNRS/INSU, BP 89, 33270 Floirac, France

7 Departamento de Física Teórica y del Cosmos, Universidad de Granada, Campus Fuentenueva, Granada, Spain

8 Instituto de Astronomía, UNAM, Campus Ensenada, Mexico

9 Max-Planck-Institut für Radioastronomie (MPIfR), Auf dem Hügel 69, 53121 Bonn, Germany
10 Department of Astronomy, University of Massachusetts, Amherst, MA 01003, USA

11 Observatoire de Paris, LERMA, CNRS, 61 av. de l'Observatoire, 75014 Paris, France

12 Institut de Radioastronomie Millimétrique, 300 rue de la Piscine, 38406 Saint Martin d'Hères, France

13 Astronomy Department, King Abdulaziz University, PO Box 80203, Jeddah, Saudi Arabia

14 Leiden Observatory, Leiden University, PO Box 9513, 2300 RA Leiden, The Netherlands

15 Australia Telescope National Facility, CSIRO, PO Box 76, Epping, NSW 1710, Australia

16 Infrared Processing and Analysis Center, MS 100-22 California Institute of Technology, Pasadena, CA 91125, USA

17 Department of Astronomy \& Astrophysics, Tata Institute of Fundamental Research, Homi Bhabha Road, 400005 Mumbai, India

18 University of British Columbia Okanagan, 3333 University Way, Kelowna, BC V1V 1V7, Canada

19 Department of Astronomy, Cornell University, Ithaca, NY 14853, USA

20 Joint Astronomy Centre, 660 North A'ohoku Place, University Park, Hilo, HI 96720, USA

21 Netherlands Organization for Scientific Research, Laan van Nieuw Oost-Indie 300, NL2509 AC The Hague, The Netherlands

22 SRON Netherlands Institute for Space Research, Landleven 12, 9747 AD Groningen, The Netherlands

23 SUPA, Institute for Astronomy, University of Edinburgh, Royal Observatory, Blackford Hill, Edinburgh EH9 3HJ, UK 